\title{
Challenges Faced by an Obstetrician while Dealing with Obstetric Emergency in Cases of Placenta Previa
}

\author{
Riddhi J. Vaghani ${ }^{1}$, Krishna Rabari², Nimish C. Pandya ${ }^{3}$ \\ ${ }^{1}$ Department of Obstetrics and Gynaecology, Gujarat Adani Institute of Medical Sciences, New Bhuj, Gujarat, India. \\ ${ }^{2}$ Department of Obstetrics and Gynaecology, Gujarat Adani Institute of Medical Sciences, New Bhuj, Gujarat, India. \\ ${ }^{3}$ Department of Obstetrics and Gynaecology, Gujarat Adani Institute of Medical Sciences, New Bhuj, Gujarat, India.
}

\section{ABSTRACT}

\section{BACKGROUND}

Placenta previa itself is a challenge for obstetrician as it may be a fatal condition. Placenta previa is a life-threatening condition and bleeds frequently after second trimester with complaints of bleeding per vagina, mildly or severely. Incidence is $35 \%$ of antepartum haemorrhage. We wanted to determine the maternal and foetal outcome at any cost by strengthening the casualty team for emergency management of unpredictable APH and PPH by giving training for the same.

\section{METHODS}

It is a descriptive observational study was conducted among 35 patients of placenta previa who were diagnosed clinically as well as on ultrasonography, from June 2017 to August 2018 in Gujarat Adani Institute of Medical Sciences. A detailed history was taken as per fixed proforma for all pregnant women.

\section{RESULTS}

Placenta previa was common in the age group of 21-30 (71.8\%) and amongst them multiparous group of more than 5 parity (29\%). 3 cases $(9.09 \%)$ were bilateral uterine artery ligation and 1 case of B-Lynch suture. A single case of internal iliac artery ligation and obstetric hysterectomy was observed. In this study 3 cases of placenta accreta, 1 case of placenta increta, 15 cases of preterm baby, 5 cases $(14.2 \%)$ of IUFD and 7 cases (20\%) of NICU admission were studied.

\section{CONCLUSIONS}

Placenta previa is a challenging job. A proper multidisciplinary approach based management including expert obstetricians, anaesthetists, neonatologists, radiologists, surgeons as well as trained nursing staff is required for saving mother's as well as baby's life.

\section{KEY WORDS}

Placenta Previa, Obstetric Emergency, Maternal and Neonatal Outcome, Team Work, Challenge

\section{Corresponding Author:} Dr. Riddhi J. Vaghani, C-302, Sai Avenue, Opp. Utran Power Station,

VIP Circle, Utran, Surat-394105, Gujarat, India.

E-mail: riddhivaghani1992@gmail.com

DOI: $10.14260 / \mathrm{jemds} / 2020 / 363$

Financial or Other Competing Interests. None.

How to Cite This Article:

Vaghani RJ, Rabari $K$, Pandya NC Challenges faced by an obstetrician while dealing with obstetric emergency in cases of placenta previa. J. Evolution Med. Dent. Sci. 2020;9(21):1654-1658, DOI:
Submission 23-08-2019,

Peer Review 16-01-2020,

Acceptance 22-01-2020,

Published 25-05-2020.

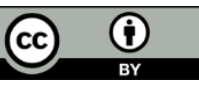




\section{BACKGROUND}

Placenta Previa in simple words means "reaching up in lower segment and further descent of placenta, causing more and more troubles". ${ }^{1}$ Placenta previa is an obstetric emergency as it is life-threatening for pregnant women as well as their babies. Classically, it presents as painless vaginal bleeding in the third trimester secondary to an abnormal placentation near or covering the internal cervical os. Placenta previa and coexistent accrete syndromes contribute maternal and perinatal morbidity and mortality rates.2,3,4

The process of "trophotropism" in which placenta grows and migrates towards best blood supply in uterus. ${ }^{5}$ The incidence of placenta previa depends on the gestational age. It is much higher before third trimester which may exceed $5 \%$ but however out of these $90 \%$ cases resolves. Incidence of placenta previa at term in various observational studies has ranged from approximately 1 in 200 to 1 in 400 . And which is still rising due to increase rate of caesarean section. ${ }^{6}$ The incidence of placenta previa increta is $2 \%$ in patient of previous one LSCS, 11\% Previous 2 LSCS, $40 \%$ previous 3 LSCS and $65 \%$ previous 4 LSCS. It also depends upon the previous pregnancy with history of placenta previa. The incidence of recurrence rate is 23 per 1000 pregnancy. The maternal mortality ratio is increased approximately threefold for women with a placenta previa. ${ }^{7}$ Management of placenta previa depends on certain factors like clinical presentation, gestational age, previous D\&C, severity of bleeding and its degree of previa and once it is confirmed action should be swift and quick.

Preterm delivery is also the major cause of perinatal death even with expectant management of placenta previa. Although some investigators suggested that congenital malformations are increased with previa. In cases of placenta previa certain study were reported that foetal anomalies were increased 2.5 fold. A team of obstetrician, anaesthetists, neonatologist, radiologist, blood bank and good nursing care can save the mother. Managing a patient of placenta previa with multidisciplinary approach will make her survival possible at the best.

Placenta previa itself is a challenge for obstetrician as it may be a fatal condition. Patient with profuse bleeding per vagina from placental site is itself a reason for maternal and foetal mortality. A team which includes Obstetrician, anaesthetist, neonatologist, radiologist, surgeons and blood bank have to work as a team and remain alert in emergency condition for saving life of a mother and the foetus.

\section{METHODS}

It was a Descriptive Observational study and conducted at G.K. General Hospital from June 2017 to August 2018. We observed 35 antepartum patients with placenta previa diagnosed at or after admission and during caesarean section. Incidence of placenta previa is 4-5/1000 pregnancy. We noted her age, parity and took detailed history of symptoms like warning bleeding episodes in earlier trimester, previous history of LSCS, previous history of D \& E, mode of delivery, distance of placenta from internal os, nature of anaesthesia like spinal or general anaesthesia, need for blood transfusion, any extra surgical intervention requiring during operative delivery like bilateral uterine artery ligation, B -lynch suture, internal iliac ligation and obstetric hysterectomy. ${ }^{8}$ For newborn history of gestational age at the time of delivery, need for NICU admission, preterm birth, asphyxiation, IUFD and presence of congenital anomaly were taken. Both mother and baby were managed till discharge from hospital.

It was approved by ethical committee of Gujarat Adani Institute of Medical Sciences. All cases were diagnosed clinically and/or by ultrasonography. Data obtained was analysed through appropriate statistical tool.

\section{Statistical Analysis}

Data analysis was performed using Statistical Package for the Social Sciences (SPSS) version 20. Mean with respective standard deviation was used to summarize a normally distributed maternal age and frequencies with respective percentages were used to summarize categorical variables. A value of less than 0.05 was considered statistically significant.

\section{RESULTS}

\section{Incidence of Placenta Previa According to Maternal Age}

Maximum incidence (74\%) between 20-34 years but in our district we find 6 patients beyond 35 years of age as their religion inhibits them from use of contraception.

\begin{tabular}{|c|c|}
\hline Grades of Placenta & No. of Patients \\
\hline$>2 \mathrm{~cm}$ away from os & 7 \\
\hline $1.1-2 \mathrm{~cm}$ from os & 5 \\
\hline$<1 \mathrm{~cm}$ from os & 16 \\
\hline \multicolumn{2}{|c|}{ Table 1. Placental Site from Internal Os } \\
According to Transvaginal Sonography \\
\hline
\end{tabular}

\section{Placental Site from Internal Os According to Transvaginal Sonography}

To localize the placenta in lower segment, full bladder is necessary. There is a challenge for sonologist to measure the distance of placenta from internal os in heavily bleeding patient in emergency. There is also a need for expert radiological opinion to identify accreta, increta \& percreta. The Gold standard tool for placental accrete syndrome is magnetic resonance imaging (MRI) but as it is time consuming \& patient is in haemodynamically unstable condition due to torrential bleeding it does not play a vital role in diagnosing accrete syndrome in emergency condition. It provides best result in a case of routine antenatal patient with a history of previous caesarean section. In this study, $45.7 \%$ patient were having complete placenta previa and $20 \%$ patient were having a low lying placenta. Almost all patients were required operative procedure.

\section{Risk Factors}

$40 \%$ patients were grand multipara. $60 \%$ patients were having previous operative history and among them $80 \%$ having placenta previa with accreta and increta. 


\begin{tabular}{|c|c|}
\hline Intra Operative Procedure & No. of Patients \\
\hline B/L uterine ligation & 4 \\
\hline B lynch suture & 1 \\
\hline B/L internal iliac ligation & 1 \\
\hline Obstetric hysterectomy & 1 \\
\hline \multicolumn{2}{|c|}{ Table 2. Intraoperative Intervention during Operation } \\
\hline
\end{tabular}

\section{Operative Challenge for Obstetrician}

Placenta previa is an uncertain condition for obstetrician as after undertaking laparotomy in previous LCSC patients there may be chances of bladder involvement as placental tissue invades and extends outside the serosa up to bladder in placenta percreta and may be adherent to abnormal site as in placenta accrete or may be invading the myometrium as in placenta increta. In caesarean section once when the baby is delivered, along with placenta, closure is never a problem but controlling the bleeding in placenta previa is a challenging job as placenta is in the lower uterine segment. There was continuous bleeding in 4 patients who required bilateral uterine artery ligation and 1 patient required B-lynch suture for the same. Patient with Placenta increta required internal iliac artery ligation leading to obstetric hysterectomy. Thus management varies from patient to patient.

\section{Challenge to Anaesthetist}

\begin{tabular}{|c|c|}
\hline Type of Anaesthesia & No. of Patients \\
\hline Spinal anaesthesia & 17 \\
\hline General anaesthesia & 18 \\
\hline \multicolumn{2}{|c|}{ Table 3. Types of Anaesthesia } \\
\hline
\end{tabular}

The anaesthetic management plan will be determined by both maternal and foetal considerations. The choice of anaesthesia is very important considering the outcome of the mother and foetus. When the patient is haemodynamically unstable, general anaesthesia is usually preferred. More number of patients require general anaesthesia because of anaemia, blood loss and shock. Not a single mortality was reported in our study.

\section{Challenge for Neonatologist}

\begin{tabular}{|c|c|}
\hline Live Baby & No. of Cases \\
\hline Preterm live Baby & 13 \\
\hline Preterm died Baby & 2 \\
\hline Asphyxiated Baby & 9 \\
\hline IUFD BABY & $5(20 \%)$ \\
\hline Table 4. Outcome in Neonates \\
\hline
\end{tabular}

In all $84 \%$ babies were alive and amongst them $36 \%$ were preterm. All babies were sent to NICU in view of birth asphyxia. $14 \%$ were born dead. Amongst them 2 babies were anomalous. Preterm infants of mothers with placenta previa were associated with a higher incidence of RDS with worse severity.

McShane et $\mathrm{al}^{9,10}$ reported that the circumstances at the time of delivery while bleeding per vagina or foetal distress predisposed the infants to anaemia, hypoxia or both which accounted for the high incidence of RDS.

\begin{tabular}{|c|c|c|}
\hline No. of Units & No. of Patients & No. of Units \\
\hline 1 unit & 18 & 18 \\
\hline 2 units & 12 & 24 \\
\hline$>3$ units & 5 & 17 \\
\hline \multicolumn{2}{|c|}{ Table 5. No. of Transfusions of Blood Products } \\
\hline
\end{tabular}

\section{Challenge for Blood Transfusion}

Out of 35 patients only 1 patient did not required blood transfusion. And rest all patients required blood transfusion. Timely availability of blood transfusion at time operation is a challenge for blood bank. There should be an agreement for blood bank to ensure rapid delivery of blood products once the massive obstetric emergency rises \& protocol is initiated.11,12,13,14 Transfusion of packed red cells should occur early and group-specific blood used until cross-matched blood is available. There is an immediate access to at least two units of O-Rhesus negative blood and more should be available. Coagulation profile helps to assess the adequacy of treatment and guide the use of blood products like Fresh Frozen Plasma (FFP) cryoprecipitate.

\section{DISCUSSION}

Several factors like gravidity, parity, number of uterine cavity operations and abortions and previous caesarean section these all influence the placental attachment site in placenta previa. Age did not influence the site of placental attachment but all above factors are associated with a greater possibility of placental attachment to the anterior wall. ${ }^{15}$ There were no differences in the rate of PAS disorders or the incidence of complete placenta previa between the anterior and posterior walls.

In the last trimester of pregnancy lower segment forms by unfolding the isthmus of the uterus. In normal pregnancy the placenta overlies the upper segment of uterus. If the placenta does overlie the lower segment, as is the case with placenta previa, it may shear off during formation of lower segment and a small section may bleed. Women with placenta previa often present with painless, bright red vaginal bleeding. This commonly occurs around 32 weeks of gestation, but can occur as early as late mid-trimester. This bleeding often starts mildly and may increase as the area of placental separation increases. Previa should be suspected if there is bleeding after 24 weeks of gestation.

Diagnosis is by history which reveals antepartum haemorrhage. Abdominal examination usually finds the uterus nontender, soft, and relaxed. Leopold's manoeuvres may find the foetus in an oblique or breech position or lying transverse as a result of the abnormal position of the placenta. Malpresentation is found in about $35 \%$ cases. Vaginal examination is avoided in known cases of placenta previa.

Previa can be confirmed by an ultrasound. Transvaginal ultrasound has superior accuracy as compared to transabdominal one, thus allowing measurement of distance between placenta and cervical os. ${ }^{16}$ However, site of placenta previa significantly influenced the pregnancy outcome; placental attachment to the anterior wall was associated with shorter gestational age, low birth weight, lower Apgar score, higher prenatal bleeding rate, increased postpartum haemorrhage, longer duration of hospitalization and higher blood transfusion and hysterectomy rates compared to cases with placental attachment to the posterior wall.

Patients with a low-lying placenta, placenta lies greater than $2 \mathrm{~cm}$ from the cervical os, may qualify for a trial of labour 
but are at an increased risk for postpartum haemorrhage and emergent caesarean section compared to women with normal placentation.17,18,19 There is no consensus for the recommended mode of delivery for marginal placenta previa. One study by Jansen et al. showed that if the distance of the edge of the placenta to the internal os is greater than 10 $\mathrm{mm}$ a trial of labour should be the recommended procedure.

A vertical skin incision is the recommended incision for optimal exposure. A high vertical uterine incision may be required if the placenta is covering the lower uterine segment or if the lower uterine segment is underdeveloped. After delivery of the foetus, the placenta spontaneously detaches, and the uterine incision can be closed. After detachment of the placenta haemorrhage will be developed secondary to the decreased contractibility of the lower uterine segment, which can be managed with bimanual uterine massage, uterotonics, intrauterine tamponade using balloon or gauze, B-Lynch sutures, Cho sutures, uterine artery or internal iliac artery ligation and uterine artery or internal iliac artery embolization. ${ }^{20,21}$ At times the massive haemorrhage may not be controlled with conservative measures and a hysterectomy is necessary. The patients with PAS and those who desires for fertility there is an option for conservative management. The placenta can be left in situ until there is devascularisation of the placental bed so that the remaining placental tissue may either be more safely removed or resorbs itself.

Also there is a threefold to fourfold increased neonatal mortality and morbidity rate with placenta previa primarily from preterm delivery. ${ }^{22}$ The neonate is at increased risk of preterm birth, lower birth weight, lower APGAR scores and increased risk for respiratory distress syndrome. Role of neonatologist is equally important for outcome in neonates in case of placenta previa. ${ }^{23,24}$

\section{CONCLUSIONS}

Placenta previa can lead to serious consequences and requires immediate attention to the presentation of vaginal bleeding. A multidisciplinary team approach must be taken to provide the utmost care for the patient.

Successful management of massive obstetric haemorrhage in an emergency situation like placenta previa, requires team work. Timely involvement of senior obstetricians, anaesthetists, haematologists, radiologist, neonatologists and nursing staff, plays a vital role. Obstetrician units should have protocols regarding major obstetric haemorrhage. Regular departmental 'mock drills' are recommended ${ }^{1}$. They help the team in familiarising with protocols and identifying deficiencies within systems. Courses such as 'Managing Obstetric Emergencies and Trauma' (MOET) and 'Advanced Life Support in Obstetrics' (ALSO) are useful.

\section{Recommendations}

Another challenge for an obstetrician is to create awareness in people regarding using government programmes for birth spacing, use of contraception to prevent multiparity and routine antenatal care in cases of placenta previa. Counselling regarding elective cesarean section, requirement of blood products during delivery and operation, and also explaining the high risk due to APH is a necessity. By undertaking such measures, we can create best possible ways to save two lives.

\section{REFERENCES}

[1] Report on Confidential Enquiries into Maternal Deaths in the United Kingdom 1997-1999. London: Royal College of Obstetricians and Gynaecologists 2001.

[2] McShane PM, Heyl PS, Epstein MF. Maternal and perinatal morbidity resulting from placenta previa. Obstet Gynecol 1985;65(2):176-82.

[3] Kassem GA, Alzahrani AK. Maternal and neonatal outcomes of placenta previa and placenta accreta: three years of experience with a two-consultant approach. Int J Womens Health 2013;5:803-10.

[4] Berhan Y. Predictors of perinatal mortality associated with placenta previa and placental abruption: an experience from a low income country. J Pregnancy 2014;2014:307043.

[5] Jung EJ, Cho HJ, Byun JM, et al. Placental pathologic changes and perinatal outcomes in placenta previa. Placenta 2018;63:15-20.

[6] Fan D, Wu S, Liu L, et al. Prevalence of antepartum hemorrhage in women with placenta previa: a systematic review and meta-analysis. Sci Rep 2017;7:40320.

[7] Pan XY, Wang YP, Zheng Z, et al. A marked increase in obstetric hysterectomy for placenta accreta. Chin Med J (Engl) 2015;128(16):2189-93.

[8] Asıcıoglu O, Şahbaz A, Güngördük K, et al. Maternal and perinatal outcomes in women with placenta praevia and accreta in teaching hospitals in Western Turkey. Journal of Obstetrics and Gynaecology 2014;34(6):462-6.

[9] Kollmann M, Gaulhofer J, Lang U, et al. Placenta praevia: incidence, risk factors and outcome. The Journal of Maternal-Fetal \& Neonatal Medicine 2016;29(9):1395-8.

[10] Schneiderman M, Balayla J. A comparative study of neonatal outcomes in placenta previa versus cesarean for other indication at term. The Journal of Maternal-Fetal \& Neonatal Medicine 2013;26(11):1121-7.

[11] Paterson-Brown S. Obstetetric emergencies. In: Edmunds DK, edr. Dewhurst Textbook of Obstetrics and Gynaecology for postgraduates. UK: Blackwell Science 2007: p. 149-52.

[12] Bener A, Saleh NM, Yousafzai MT. Prevalence and associated risk factors of ante-partum hemorrhage among Arab women in an economically fast growing society. Niger J Clin Pract 2012;15(2):185-9.

[13] Agboola A. Antepartum haemorrhage. In: Textbook of Obstetrics and Gynaecology for medical students. Lagos: Heinemann Education Books Publication 2006: p. 340-7.

[14] Mukherjee S, Bhide A. Antepartum haemorrhage. Obstet Gynaecol Reprod Med 2008;18(12):335-9.

[15] Koken GN, Kanat-Pektas M, Köse SK, et al. Maternal blood pressure and dominant sleeping position may affect placental localization. The Journal of Maternal-Fetal \& Neonatal Medicine 2014;27(15):1564-7.

[16] Durst JK, Tuuli MG, Temming LA, et al. Resolution of a lowlying placenta and placenta previa diagnosed at the 
midtrimester anatomy scan. Journal of Ultrasound in Medicine 2018;37(8):2011-9.

[17] Allahdin S, Voigt S, Htwe TT. Management of placenta previa and accreta. J Obstet Gynaecol 2011;31(1):1-6.

[18] Miller DA, Chollet JA, Goodwin TM. Clinical risk factors for placenta previa-placenta accreta. Am J Obstet Gynecol 1997;177(1):210-4.

[19] Sekiguchi A, Nakai A, Kawabata I, et al. Type and location of placenta previa affect preterm delivery risk related to a tepartum hemorrhage. Int J Med Sci 2013;10(2):1683-8.

[20] Sallam HF, Shady NW. A sandwich technique (N\&H variation technique) to reduce blood loss during cesarean delivery for complete placenta previa: a randomized controlled trial. The Journal of Maternal-Fetal \& Neonatal Medicine 2019;32(19):3145-52.
[21] Ryu JM, Choi YS, Bae JY. Bleeding control using intrauterine continuous running suture during cesarean section in pregnant women with placenta previa. Archives of Gynecology and Obstetrics 2019;299(1):135-9.

[22] Dola CP, Garite TJ, Dowling DD, et al. Placenta previa: does its type affect pregnancy outcome? Am J Perinatal 2003;20(7):353-60.

[23] Matsuda Y, Itoh T, Itoh $\mathrm{H}$, et al. Impact of placental weight and fetal/placental weight ratio $\mathrm{Z}$ score on fetal growth and the perinatal outcome. Int J Med Sci 2018;15(5):48491.

[24] Jain S, Jain N, Dahiya P, et al. Perinatal outcome in antepartum hemorrhage in teaching hospital of northern India - a prospective study. International Journal of Scientific and Research Publications 2015;5(2):1-6. 